\title{
Emerging Endoscopic and Photodynamic Techniques for Bladder Cancer Detection and Surveillance
}

\author{
Prashant Patel, ${ }^{1,2}$ Richard T. Bryan, ${ }^{1}$ and D. Michael A. Wallace ${ }^{2}$ \\ ${ }^{1}$ School of Cancer Sciences, University of Birmingham, Edgbaston B15 2TT, UK \\ ${ }^{2}$ Department of Urology, QE Hospital Birmingham, Edgbaston B15 2TH, UK
}

Received 1 August 2011; Accepted 6 December 2011

Academic Editor: Andrew Karim Kader

This review provides an overview of emerging techniques, namely, photodynamic diagnosis (PDD), narrow band imaging (NBI), Raman spectroscopy, optical coherence tomography, virtual cystoscopy, and endoscopic microscopy for its use in the diagnosis and surveillance of bladder cancer. The technology, clinical evidence and future applications of these approaches are discussed with particular emphasis on PDD and NBI. These approaches show promise to optimise cystoscopy and transurethral resection of bladder tumours.

KEYWORDS: bladder, cancer, diagnosis, surveillance, fluorescence, cystoscopy, narrow, band, imaging, optical, coherence, tomography, photodynamic, diagnosis, Raman, spectroscopy, virtual, cystoscopy, endoscopic, microscope, urothelial, cell, carcinoma 


\section{INTRODUCTION}

Bladder cancer is the ninth most common cancer in the world, affecting more than 356,600 people each year. It has the highest incidence in Egypt followed by Europe and North America [1]. The majority of diagnosed patients (75-85 percent) present with nonmuscle invasive bladder cancer (NMIBC), which is characterised by a probability of recurrence at 1 and 5 years of 15-61 and 31-78 percent, respectively. Moreover, progression of disease is seen in this group from $<1-17 \%$ and $<1-45 \%$ at 1 and 5 years, respectively [2].

Flexible cystoscopy and voided urine cytology are currently the initial investigations of choice for patients with symptoms suggestive of bladder cancer. The mainstay of treatment for NMIBC is complete transurethral resection (TUR), ultimately preventing disease recurrence and progression. It is postulated that bladder cancer recurrence occurs via four mechanisms: incomplete resection, tumour cell reimplantation, growth of microscopic tumours, and new tumour formation [3]. First tumour recurrence appears different to subsequent recurrences; incomplete resection and tumour cell reimplantation may dominate at this timepoint and are therefore influenced by clinicians before and immediately after resection [4]. Only later does genuine new tumour formation appear to increase in importance where chemopreventive agents may have its role in reducing recurrence.

Nonmuscle invasive urothelial transitional cell cancer (UC) of the bladder is one of the most expensive cancers to manage on a per patient basis, because of its high prevalence, high recurrence rate, and the need for long-term cystoscopic surveillance. The total cost of treatment and 5-year followup of patients with NMIBC diagnosed during 2001-2002 in the United Kingdom was over £35 million [5]. Direct cost of managing bladder cancer in the USA is estimated to range from $\$ 96,000-\$ 187,000$ per patient from the diagnosis to death [6]. Furthermore, health technology assessments have demonstrated the addition of photodynamic diagnosis (PDD) and urinary biomarkers adds to the cost which can have implications towards service provision $[7,8]$.

Developments in optical diagnostics might reduce the limitations of current methods of detection and surveillance in several ways. These emerging techniques aim at cystoscopy for better visualisation of bladder tumours or predict histopathologic diagnosis in realtime. We aim to review these relatively new technologies for their ability to improve the diagnostic yield.

\section{METHODS}

A PubMed literature search was performed for this nonsystematic review, and papers on photodynamic diagnosis (PDD), narrow-band imaging (NBI), Raman spectroscopy (RS), optical coherence tomography (OCT), virtual cystoscopy (VC), and endoscopic-microscope (EM) regarding bladder cancer were reviewed.

\section{PHOTODYNAMIC DIAGNOSIS (PDD)}

PDD is a technique that has been proposed to enhance tumour detection and resection. The principle of PDD is based on the interaction between a photosensitising agent with a high uptake by tumour cells and light with an appropriate wavelength, which is absorbed by the agent and reemitted with a different wavelength [9].

Several commercially available agents have been used to induce exogenous fluorescence, but presently hypericin, 5 aminolaevulinic acid (5-ALA), and its ester hexaminolevulinate (HAL) are applied most often. Optimal dose and instillation time have not yet been determined. One retrospective trial compared the performance of PDD with HAL and with 5-ALA [10]. No significant differences were found between 5-ALA and HAL in this study. A randomised comparison of both substances has never been performed. 
Several clinical trials have shown that detection of bladder cancer with PDD is superior to white light cystoscopy (WLC). In a systematic review and meta-analysis, twenty-seven studies (2,949 participants) were assessed for test performance [11]. PDD had higher sensitivity than WLC (92\%, 95 percent confidence interval [CI], 80-100\% versus $71 \%$, 95 percent CI, 49-93\%) but lower specificity (57\%, 95 percent CI, 36$79 \%$ versus $72 \%, 95$ percent CI, $47-96 \%$ ).

In order to predict short- and long-term risks of recurrence and progression, the EORTC-GU group has developed a scoring system and risk table based on number of tumours, tumour size, prior recurrence rate, T-category, presence of concurrent CIS, and tumour grade into low, intermediate, and high risk groups [2]. For detecting higher-risk tumours, median range sensitivity of PDD (89\%, 95 percent CI, 6-100\%) was higher than WLC (56\%, 95 percent CI, 0-100\%) whereas for lower-risk tumours, it was broadly similar $(92 \%, 95$ percent CI, 20-95\%) versus 95\%, (95 percent CI, 8-100\%). The higher sensitivity of PDD was also reflected in the detection of CIS alone. Four randomised clinical trials (RCTs) involving 709 participants using 5 aminolaevulinic acid (5-ALA) as the photosensitising agent reported clinical effectiveness. Using PDD at transurethral resection of bladder tumour (TURBT) resulted in fewer residual tumours at check cystoscopy (relative risk [RR], 0.37, 95 percent CI, 0.20-0.69) and longer recurrence-free survival (RR, 1.37, 95 percent CI, 1.18-1.59), compared with WLC. However, the advantages of PDD at TURBT in reducing recurrence and progression in the longer term were less clear [8].

A recent study examined the frequency of HAL-fluorescence-detected residual tumours immediately after standard WL TURBT and the efficacy of immediate removal of residual tumour tissue or overlooked tumours on tumour recurrence. Patients were first inspected and resected with white light before undergoing inspection and further resection with blue light HAL fluorescence cystoscopy. It was therefore possible not only to identify previously overlooked lesions, but also to identify areas of residual tumour where the resection under white light had not been complete. Fluorescence-guided cystoscopy after complete WL TURBT identified residual tumour tissue in 44 of 90 patients (49\%). The recurrence rate in patients followed for 12 months was $47.3 \%$ after WL TURB and 30.5\% after HAL TURBT $(P=0.05)$ [12]. This study also demonstrates the potential of using PDD TURB as a teaching tool to offer clear visualisation of tumours and their margins, thereby facilitating improved TURBT.

The European expert panel recommended HAL-guided PDD use on all patients with initial suspicion of bladder cancer [13]. However, a recent cost analysis demonstrated that PDD added a high cost to benefit ratio if applied to all patients in detection and surveillance of bladder cancer [8]. Nonetheless, PDD may be cost effective in selected group with high-risk disease for surveillance. This remains to be determined. Furthermore, PDD may have its role in patients with positive urine cytology but negative WLC.

\section{NARROW-BAND IMAGING (NBI)}

NBI is an optical image enhancement technique designed for endoscopy to enhance the contrast between mucosal surfaces and microvascular structures without the use of dyes. This narrow band of light is strongly absorbed by haemoglobin and penetrates only the surface of tissue, increasing the visibility of capillaries and other delicate tissue surface structures [14].

Level I evidence (meta-analysis) of NBI use is lacking in bladder cancer [15] but does exist in the field of gastro-enterology for detection of colonic adenoma [16], high-grade dysplasia and metaplasia in Barrett's oesophagus [17].

There are few studies published assessing the value of NBI in bladder cancer. All found a subjective improvement of visualisation of the tumours. Bryan et al. performed flexible WLC and subsequent NBI cystoscopy in 29 patients with recurrent NMIBC. NBI cystoscopy revealed 15 additional tumours in 12 patients [14]. However, the additional tumours were not confirmed with histology since all tumours were treated with diathermy ablation. In a further study involving 23 patients by the same group demonstrated that even "new users" to NBI technology demonstrate a significantly improved detection rate of bladder cancer using NBI versus WLC alone [18]. Herr et al. performed WLC with subsequent NBI cystoscopy in 427 consecutive patients with a history of NMIBC. Recurrence was found in 103 patients. In 56\% of patients 
with a recurrence $(n=58)$, additional tumours were detected by NBI, and in $12 \%$ of patients $(n=13)$, the bladder tumours were detected only by NBI.

A prospective controlled study of NBI was conducted in 104 consecutive patients with definite or suspected bladder cancer by Tatsugami and colleagues [19]. They reported a sensitivity and specificity for the detection of bladder tumours using NBI in all patients versus those with CIS of $92.7 \%$ and 70.9 versus $89.7 \%$ and $74.5 \%$, respectively. The sensitivity and specificity for the detection of bladder tumours using NBI in patients with positive versus negative urine cytology were $85.4 \%$ versus $98.4 \%$ and $75.7 \%$ versus $66.3 \%$, respectively. For WLC and NBI cystoscopy, the overall sensitivity was $87 \%$ and $100 \%$ and the overall specificity $85 \%$ and $82 \%$, respectively [20]. A limitation of this study is the possible observer bias, since WLC and NBI were performed subsequently by the same urologist. In order to address observer bias a recent randomized trial confirmed that a "second look" did not compromise the superiority of NBI over standard WLI flexible cystoscopy for detecting primary NMIBC including CIS lesions [38]. Whether the specificity of NBI will be negatively influenced by previous intravesical instillations, inflammation, or scarring, like PDD, is yet unknown.

Reassuringly, NBI cystoscopy does not appear to have a "learning curve" for its adaptation in surveillance for patients with bladder cancer [21].

\section{RAMAN SPECTROSCOPY (RS)}

RS enables measurement of molecular components of tissue in a qualitative and quantitative way. The principle of this optical technique is based on the Raman effect or inelastic scattering. Raman molecular imaging (RMI) is an optical technology that combines the molecular chemical analysis of Raman spectroscopy with high-definition digital microscopic visualisation [9]. This approach permits visualisation of the physical architecture and molecular environment of cells in the urine. The Raman spectrum of a cell is a complex product of its chemical bonds.

Several groups have determined the diagnostic accuracy of RS by comparing ex vivo Raman measurements of bladder samples with histology. Generally, the time needed to obtain the spectra is between 1 and 5 seconds [22]. Shapiro and colleagues investigated urine samples from 340 patients, including 116 patients without UC, 92 patients with low-grade tumors, and 132 patients with high-grade tumours. The Raman spectra from UC tissue demonstrate a distinct peak at a $1584 \mathrm{~cm}^{-1}$ wave shift not present in benign tissues. The height of this peak correlated with the tumour's grade. The signal obtained from epithelial cells correctly diagnosed bladder cancer with sensitivity of $92 \%$ (100\% of the high-grade tumors), specificity of $91 \%$, a positive predictive value of $94 \%$, and a negative predictive value of $88 \%$. The signal correctly assigned a tumour's grade in $73.9 \%$ of the low-grade tumors and $98.5 \%$ of the high-grade tumours [23].

RMI for diagnosis of bladder cancer is limited by the need for specialised equipment and training of laboratory personnel. For in vivo measurements, small, flexible fibre-optic probes compatible with the working channel of a rigid or flexible cystoscope have been developed [22], but the application has been hampered by many technical issues [9]. Therefore, further studies demonstrating human in vivo applicability of RS on bladder tissue are still awaited.

\section{OPTICAL COHERENCE TOMOGRAPHY (OCT)}

OCT produces high-resolution, cross-sectional images of tissue. The principle of this optical technique is analogous to B-mode ultrasonography except that light is being used instead of sound [24].

Karl et al. enrolled 52 patients who underwent transurethral bladder biopsy or TUR-BT for surveillance or due to initial suspicion of UC of the bladder. In total, 166 lesions were suspicious for malignancy according to standard white light cystoscopy. All suspicious lesions were scanned and interpreted during perioperative cystoscopy using OCT, and then subsequently biopsied by cold cup or TUR for pathological confirmation. There were no false-negative lesions detected by OCT. Sensitivity of OCT for detecting the presence of a malignant lesion was $100 \%$ and sensitivity for detection of tumor 
growth beyond the lamina propria was $100 \%$ as well. Specificity of OCT for presence of malignancy was $65 \%$, due to the fact that a number of lesions were interpreted as false positive by OCT [25].

As a minimally invasive technique, OCT demonstrates high sensitivity for detection of malignant lesions as well as estimation of whether a tumour has invaded beyond the lamina propria [24, 26, 27]. However, specificity of OCT within the bladder is low, possibly due to a learning curve and/or the relatively low spatial resolution and visualisation depth of the OCT technology. Further studies and technical development are needed to establish an adequate surrogate for optical biopsy.

Advantages of OCT can be found in the noninvasive, real-time, and high-resolution images that are comparable with histopathology and provide information about depth of tumour growth. However, reliable measurements of muscle-invasive tumours may be hampered due to insufficient imaging depth. OCT is less suitable for screening the entire bladder, thus in the absence of visually suspect lesions, it has to be used in combination with other methods (e.g., NBI or PDD) to direct to the region of interest.

\section{VIRTUAL CYSTOSCOPY (VC)}

With the progressive development in diagnostic imaging and medical computer software technologies, it is possible to generate virtual reality images to aid the clinician to inspect the interior of the bladder in real time. VC can be applied to any imaging modality be it computerised tomography (CTVC), magnetic resonance imaging (MRVC), or ultrasound (USVC).

A metaanalysis comparing virtual cystoscopy and ultrasonography (US) was reported by Qu and colleagues [28]. A total of 26 studies that included 3084 patients who fulfilled all of the inclusion criteria were considered for inclusion in the analysis. The pooled sensitivity for bladder cancer detection using CT virtual cystoscopy (CTVC), MR virtual cystoscopy (MRVC), and US was 0.939 (95\% CI, 0.919-0.956), 0.908 (95\% CI, 0.827-0.959), and 0.779 (95\% CI, 0.744-0.812), respectively. The pooled specificity for bladder cancer detection using CTVC, MRVC, and US was 0.981 (95\% CI, 0.973-0.988), 0.948 (95\% CI, 0.884-0.983), and 0.962 (95\% CI, 0.953-0.969), respectively. The pooled diagnostic odd ratio (DOR) estimate for CTVC (604.22) was significantly higher than for MRVC $(144.35, P<0.001)$ and US (72.472, $P<0.001)$. Results showed that both CTVC and MRVC are better imaging methods for diagnosing bladder cancer than US. CTVC has higher diagnostic value (sensitivity, specificity, and DOR) for the detection of bladder cancer than either MRCT or US.

CT virtual cystoscopy is a noninvasive technique that can be used successfully for detection of bladder tumours $>5 \mathrm{~mm}$ in selected cases during daily routine abdominopelvic work. UCs $>5 \mathrm{~mm}$ represent the majority of newly diagnosed bladder tumours: in the Bladder Cancer Prognosis Programme [29], only $6.7 \%$ of 1075 confirmed UCs were $5 \mathrm{~mm}$ or less in size (unpublished data). In some series, lesions as small as $2 \times 3 \mathrm{~mm}$ have been detected [30], but this technique is still unable to detect flat lesions that may represent CIS [31]. Moreover, the effects of ionising radiation are a significant obstacle to such modalities being used routinely for diagnosis and surveillance of bladder cancer.

\section{ENDOSCOPIC MICROSCOPE (EM)}

EM is a novel, low-cost, high-resolution endoscopic microscope for obtaining fluorescent images of the cellular morphology of the epithelium. Its experimental use as a noninvasive point imaging system offers a method for obtaining real-time histologic information during endoscopy [32]. Whilst it is feasible to obtain high-resolution histopathologic information using the endoscopic microscope device, the difficulties of holding a fine (1.4-2.6 mm in diameter) instrument still to capture the image within the bladder makes obtaining interpretable images challenging. Future improvement and integration with wide field endoscopic techniques will aid in improving the sensitivity of detection of dysplasia and early cancer development in epithelial cancers like the oesophagus or bladder. 
TABLE 1: Overview of NBI and PDD techniques.

\begin{tabular}{|c|c|c|}
\hline & NBI & PDD \\
\hline Optical principle & Absorption & Fluorescence \\
\hline Costs stack & Equivalent & Equivalent \\
\hline Costs scopes & $\begin{array}{l}\text { Can be used on compatible } \\
\text { "scopes" }\end{array}$ & $\begin{array}{l}\text { New rigid "scopes" with blue light filter } \\
\text { are required }\end{array}$ \\
\hline Costs staff & Nil & Extra \\
\hline Costs consumables & Nil & Extra \\
\hline Convenience & Very convenient & Inconvenient \\
\hline Extra invasiveness for patient & None & $\begin{array}{l}\text { Extra catheterisation for preoperative } \\
\text { instillation of photosensitising agent }\end{array}$ \\
\hline $\begin{array}{l}\text { Sensitivity (\%) for visualisation of } \\
\text { bladder tumours }\end{array}$ & $93-100$ & $82-97$ \\
\hline $\begin{array}{l}\text { Specificity (\%) for visualisation of } \\
\text { bladder tumours }\end{array}$ & $68-82$ & $41.4-98.5$ \\
\hline $\begin{array}{l}\text { On Initial suspicion of bladder cancer } \\
\text { diagnosis }\end{array}$ & $\begin{array}{l}\text { Good in an outpatient setting at } \\
\text { initial cystoscopy }\end{array}$ & $\begin{array}{l}\text { Good in an inpatient setting at time of } \\
\text { TURBT }\end{array}$ \\
\hline On CIS diagnosis & Accuracy of $83 \%[38]$ & Good \\
\hline $\begin{array}{l}\text { In patients with positive urine } \\
\text { cytology but negative WLC }\end{array}$ & Not known & Recommended \\
\hline $\begin{array}{l}\text { For assessment at time of tumour } \\
\text { recurrences in patients not previously } \\
\text { staged with NBI/PDD }\end{array}$ & Not known & Recommended \\
\hline Tumour treatment & $\begin{array}{l}\text { Good in outpatient setting for } \\
\text { ablation of tumours by diathermy } \\
\text { or laser, especially for } \\
\text { recurrences during surveillance }\end{array}$ & $\begin{array}{l}\text { Good in an inpatient setting in } \\
\text { conjunction with TURBT }\end{array}$ \\
\hline Haematuria clinic & Considerable potential & $\begin{array}{l}\text { Impractical due to patient numbers and } \\
\text { not all patients presenting to haematuria } \\
\text { clinic have UC }\end{array}$ \\
\hline For surveillance & $\begin{array}{l}\text { Good. Effect of previous BCG, } \\
\text { inflammation or scarring is yet } \\
\text { unknown }\end{array}$ & $\begin{array}{l}\text { No data available. Specificity is reduced } \\
\text { in patients who have had previous BCG, } \\
\text { inflammation, or scars and hence limits } \\
\text { the use }\end{array}$ \\
\hline Extra applications & $\begin{array}{l}\text { Upper urinary tract, upper and } \\
\text { lower GI, and so forth }\end{array}$ & Nil \\
\hline As a teaching tool & Good & Good \\
\hline
\end{tabular}

\section{CONCLUSION}

As described earlier, the 4 mechanisms of bladder UC recurrence are incomplete resection, tumour cell reimplantation, growth of microscopic tumours, and new tumour formation [3]. Incomplete resection and tumour cell reimplantation at the site of the primary tumour are proposed to be the most important causes of early UC recurrence, as well as the growth of very small volume or microscopic tumours that were present but overlooked at the time of the primary resection [3]. Improving optical technologies is one way to overcome these WLC shortfalls. In this regard, PDD and NBI are the most mature technologies and are currently in widespread clinical use. An overview of these two most studied approaches is outlined in Table 1. The other modalities described above may also facilitate the detection of very small volume or 
microscopic lesions, but their future is more likely to lie with "real-time" tumour staging. An instrument that combines some or all of these technologies may be the ultimate goal for the urologist, but this is some way off yet.

Another strategy to improve our optical diagnosis of bladder UCs is by improving cystoscopy skills. Cystoscopy may not be taught as well as it should. This may be due to poor training and the assumption that junior urologists do not need formal training in performing cystoscopy and are not assessed in this. Moreover, delegation of the large volume of followup cystoscopy is usually to junior and inexperienced staff. Also, failure to use the best endoscopy equipment and not employing the 70-degree telescope are possible reasons for poor-quality TURBTs, even amongst experienced urologists. The absence of detrusor muscle in the first apparently complete, white light TURBT by less-experienced surgeons appears to be independently associated with an increased risk of recurrence [33]. TURBT should be performed in a timely fashion and under standard conditions. These include use of proper anaesthesia with a continuous-flow video resectoscope. The entire bladder must be visualised and all abnormal areas must be resected, with separate biopsies from each tumour's base, specifically to avoid understaging muscle-invasive disease. Restaging TURBT is recommended for all high-grade tumours, particularly if the muscle is not present in the specimen [34]. The main evidence for this is supported by Herr showing the large number of tumours missed and the high recurrence rate [35]. This is thought due to inadequate training, technique, or both. Users of PDD/NBI have often reported that "these techniques make one a better cystoscopist" by focusing the operator in taking longer and looking more thoroughly. From personal experience, when we first started using NBI flexible cystoscopy in 2005, we were surprised at just how many UCs we were missing with WLC, and quickly learnt to recognise some of the very subtle mucosal changes that were visible with WLC that accompanied the lesions that we had only initially seen with NBI. Other authors describe how they now routinely resect or diathermy a margin around the base of the primary UC as a result of what they have seen when performing TURs with PDD [36].

Data from this review indicate at evidence levels 1 and 2 (metaanalysis of randomised controlled and good-quality prospective cohort studies) that PDD detects significantly more tumour-positive patients than white-light cystoscopy alone. This may come at the price of reduced specificity. The detection benefit is higher in selected patients with carcinoma in situ (evidence level 2). PDD reduces residual tumour rates and increases tumour-free survival significantly (evidence level 1). Measures to make it more cost effective include using PDD in a more selective setting such as those with high-risk disease (EORTC-GU group tables) at first diagnosis or in patients with positive urine cytology but negative WLC.

Although a clear benefit for PDD has been found for the detection of CIS, the value of this technique with respect to CIS recurrences and progression remains unclear. The benefit of PDD with respect to progression must also be demonstrated in papillary disease especially over the course of longterm surveillance. Presently no data exists on which to base a firm recommendation for the use of PDD for surveillance. Future studies will have to show if the intervals to followup cystoscopy can be altered when PDD is used and if PDD changes adjuvant treatment, for instance, due to the safe exclusion of CIS. Furthermore, the necessity of re-TUR after PDD must be evaluated.

With both NBI and PDD there remain the issues of whether the procedures are carried out more diligently when the new modality is used [36], or whether the improvements are due to a "second look" cystoscopy [37]. However, a recent study has concluded that "a second look does not compromise the superiority of NBI over standard WLC flexible cystoscopy for detecting primary NMIBC including CIS [38]."

Other techniques discussed, namely, RS, OCT, VC, and EM show promising preliminary results, but more research is needed before any of the techniques can be instituted in the diagnosis and surveillance of bladder cancer. There is clearly a need for larger, multicentre RCT in these emerging approaches, especially NBI and PDD for diagnosis and surveillance of bladder cancer. A CROES trial is currently recruiting in the NBI TUR setting, and other trials using NBI and PDD for bladder cancer surveillance are in planning. 


\section{REFERENCES}

[1] IARC, "GLOBOCAN 2002. Cancer Incidence, Mortality and Prevalence Worldwide (2002 estimates)," http:// globocan.iarc.fr/.

[2] R. J. Sylvester, A. P. M. van der Meijden, W. Oosterlinck et al., "Predicting recurrence and progression in individual patients with stage Ta T1 bladder cancer using EORTC risk tables: a combined analysis of 2596 patients from seven EORTC trials," European Urology, vol. 49, no. 3, pp. 466-475, 2006.

[3] J. Kondás, L. Kiss, A. Határ et al., "The effect of intravesical mitomycin C on the recurrence of superficial (TaT1) bladder cancer. A Hungarian multicenter study," International Urology and Nephrology, vol. 31, no. 4, pp. 451-456, 1999.

[4] R. T. Bryan, S. I. Collins, M. C. Daykin et al., "Mechanisms of recurrence of Ta/T1 bladder cancer," Annals of the Royal College of Surgeons of England, vol. 92, no. 6, pp. 519-524, 2010.

[5] V. K. Sangar, N. Ragavan, S. S. Matanhelia, M. W. Watson, and R. A. Blades, "The economic consequences of prostate and bladder cancer in the UK," BJU International, vol. 95, no. 1, pp. 59-63, 2005.

[6] M. F. Botteman, C. L. Pashos, A. Redaelli, B. Laskin, and R. Hauser, "The health economics of bladder cancer: a comprehensive review of the published literature," PharmacoEconomics, vol. 21, no. 18, pp. 1315-1330, 2003.

[7] A. M. Kamat, J. A. Karam, H. B. Grossman, A. K. Kader, M. Munsell, and C. P. Dinney, "Prospective trial to identify optimal bladder cancer surveillance protocol: reducing costs while maximizing sensitivity," $B J U$ International, vol. 108, no. 7, pp. 1119-1123, 2011.

[8] G. Mowatt, S. Zhu, M. Kilonzo et al., "Systematic review of the clinical effectiveness and cost-effectiveness of photodynamic diagnosis and urine biomarkers (FISH, ImmunoCyt, NMP22) and cytology for the detection and follow-up of bladder cancer," Health Technology Assessment, vol. 14, no. 4, pp. 1-331, 2010.

[9] E. C. C. Cauberg, D. M. de Bruin, D. J. Faber, T. G. van Leeuwen, J. J. M. C. H. de la Rosette, and T. M. de Reijke, "A new generation of optical diagnostics for bladder cancer: technology, diagnostic accuracy, and future applications," European Urology, vol. 56, no. 2, pp. 287-296, 2009.

[10] M. Burger, C. G. Stief, D. Zaak et al., "Hexaminolevulinate is equal to 5-aminolevulinic acid concerning residual tumor and recurrence rate following photodynamic diagnostic assisted transurethral resection of bladder tumors," Urology, vol. 74, no. 6, pp. 1282-1286, 2009.

[11] G. Mowatt, J. N'Dow, L. Vale et al., "Photodynamic diagnosis of bladder cancer compared with white light cystoscopy: systematic review and meta-analysis," International Journal of Technology Assessment in Health Care, vol. 27, no. 1, pp. 3-10, 2011.

[12] G. G. Hermann, K. Mogensen, S. Carlsson, N. Marcussen, and S. Duun, "Fluorescence-guided transurethral resection of bladder tumours reduces bladder tumour recurrence due to less residual tumour tissue in $\mathrm{T}$ a/T1 patients: a randomized two-centre study," BJU International, vol. 108, no. 8, pp. E297-E303, 2011.

[13] J. A. Witjes, J. P. Redorta, D. Jacqmin et al., "Hexaminolevulinate-guided fluorescence cystoscopy in the diagnosis and follow-up of patients with non-muscle-invasive bladder cancer: review of the evidence and recommendations," European Urology, vol. 57, no. 4, pp. 607-614, 2010.

[14] R. T. Bryan, L. J. Billingham, and D. M. A. Wallace, "Narrow-band imaging flexible cystoscopy in the detection of recurrent urothelial cancer of the bladder," BJU International, vol. 101, no. 6, pp. 702-705, 2008.

[15] H. H. Herr, "Narrow band imaging cystoscopy," Urologic Oncology, vol. 29, pp. 353-357, 2011.

[16] Y. Kobayashi, Y. Hayashino, J. L. Jackson, N. Takagaki, S. Hinotsu, and K. Kawakami, "Diagnostic performance of chromoendoscopy and narrow band imaging for colonic neoplasms: a meta-analysis," Colorectal Disease, vol. 14, no. 1, pp. 18-28, 2012.

[17] J. Mannath, V. Subramanian, C. J. Hawkey, and K. Ragunath, "Narrow band imaging for characterization of high grade dysplasia and specialized intestinal metaplasia in Barretts esophagus: a meta-analysis," Endoscopy, vol. 42, no. 5, pp. 351-359, 2010.

[18] R. T. Bryan, Z. H. Shah, S. I. Collins, and D. M. A. Wallace, "Narrow-band imaging flexible cystoscopy: a new user's experience," Journal of Endourology, vol. 24, no. 8, pp. 1339-1343, 2010.

[19] K. Tatsugami, K. Kuroiwa, T. Kamoto et al., "Evaluation of narrow-band imaging as a complementary method for the detection of bladder cancer," Journal of Endourology, vol. 24, no. 11, pp. 1807-1811, 2010.

[20] H. W. Herr and S. M. Donat, "A comparison of white-light cystoscopy and narrow-band imaging cystoscopy to detect bladder tumour recurrences," BJU International, vol. 102, no. 9, pp. 1111-1114, 2008. 
[21] H. Herr, M. Donat, G. Dalbagni, and J. Taylor, "Narrow-band imaging cystoscopy to evaluate bladder tumoursindividual surgeon variability," BJU International, vol. 106, no. 1, pp. 53-55, 2010.

[22] R. O. P. Draga, M. C. M. Grimbergen, P. L. M. Vijverberg et al., "In vivo bladder cancer diagnosis by high-volume Raman spectroscopy," Analytical Chemistry, vol. 82, no. 14, pp. 5993-5999, 2010.

[23] A. Shapiro, O. N. Gofrit, G. Pizov, J. K. Cohen, and J. Maier, "Raman molecular imaging: a novel spectroscopic technique for diagnosis of bladder cancer in urine specimens," European Urology, vol. 59, no. 1, pp. 106-112, 2011.

[24] S. P. Lerner, "Innovations in endoscopic imaging for bladder cancer," European Urology, vol. 56, no. 6, pp. 920-922, 2009.

[25] A. Karl, H. Stepp, E. Willmann et al., "Optical coherence tomography for bladder cancer-ready as a surrogate for optical biopsy? Results of a prospective mono-centre study," European Journal of Medical Research, vol. 15, no. 3, pp. 131-134, 2010.

[26] B. Hermes, F. Spöler, A. Naami et al., "Visualization of the basement membrane zone of the bladder by optical coherence tomography: feasibility of noninvasive evaluation of tumor invasion," Urology, vol. 72, no. 3, pp. 677-681, 2008.

[27] M. J. Manyak, N. D. Gladkova, J. H. Makari et al., "Evaluation of superficial bladder transitional-cell carcinoma by optical coherence tomography," Journal of Endourology, vol. 19, no. 5, pp. 570-574, 2005.

[28] X. Qu, X. Huang, L. Wu, G. Huang, X. Ping, and W. Yan, "Comparison of virtual cystoscopy and ultrasonography for bladder cancer detection: a meta-analysis," European Journal of Radiology, vol. 80, no. 2, pp. 188-197, 2010.

[29] M. P. Zeegers, R. T. Bryan, C. Langford et al., "The West Midlands Bladder cancer prognosis programme: rationale and design," BJU International, vol. 105, no. 6, pp. 784-788, 2010.

[30] O. R. Karabacak, E. Cakmakci, U. Ozturk et al., "Virtual cystoscopy: the evaluation of bladder lesions with computed tomographic virtual cystoscopy," Journal of the Canadian Urological Association, vol. 5, no. 1, pp. 34-37, 2011.

[31] A. R. Zlotta, "What is the future of virtual cystoscopy in urology?" Journal of the Canadian Urological Association, vol. 5, no. 1, pp. 38-39, 2011.

[32] T. J. Muldoon, S. Anandasabapathy, D. Maru, and R. Richards-Kortum, "High-resolution imaging in Barrett's esophagus: a novel, low-cost endoscopic microscope," Gastrointestinal Endoscopy, vol. 68, no. 4, pp. 737-744, 2008.

[33] P. Mariappan, A. Zachou, and K. M. Grigor, "Detrusor muscle in the first, apparently complete transurethral resection of bladder tumour specimen is a surrogate marker of resection quality, predicts risk of early recurrence, and is dependent on operator experience," European Urology, vol. 57, no. 5, pp. 843-849, 2010.

[34] A. M. Nieder and M. Manoharan, "The role of the surgeon and transurethral resection in the treatment of superficial bladder cancer," TheScientificWorldJOURNAL, vol. 6, pp. 2626-2631, 2006.

[35] H. W. Herr, "Role of re-resection in non-muscle-invasive bladder cancer," TheScientificWorldJOURNAL, vol. 11, pp. 283-288, 2011.

[36] T. O'Brien and K. Thomas, "Bladder cancer: photodynamic diagnosis can improve surgical outcome," Nature Reviews Urology, vol. 7, no. 11, pp. 598-599, 2010.

[37] E. C. C. Cauberg, S. Kloen, M. Visser et al., "Narrow band imaging cystoscopy improves the detection of nonmuscle-invasive bladder cancer," Urology, vol. 76, no. 3, pp. 658-663, 2010.

[38] Y. J. Shen, Y. P. Zhu, D. W. Ye et al., "Narrow-band imaging flexible cystoscopy in thedetection of primary non-muscle invasive bladder cancer: a "second look" matters?" International Urology and Nephrology. In press.

\section{This article should be cited as follows:}

Prashant Patel, Richard T. Bryan, and D. Michael A. Wallace, "Emerging Endoscopic and Photodynamic Techniques for Bladder Cancer Detection and Surveillance," TheScientificWorldJOURNAL, vol. 11, pp. 2550-2558, 2011. 


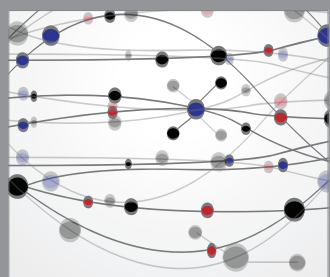

The Scientific World Journal
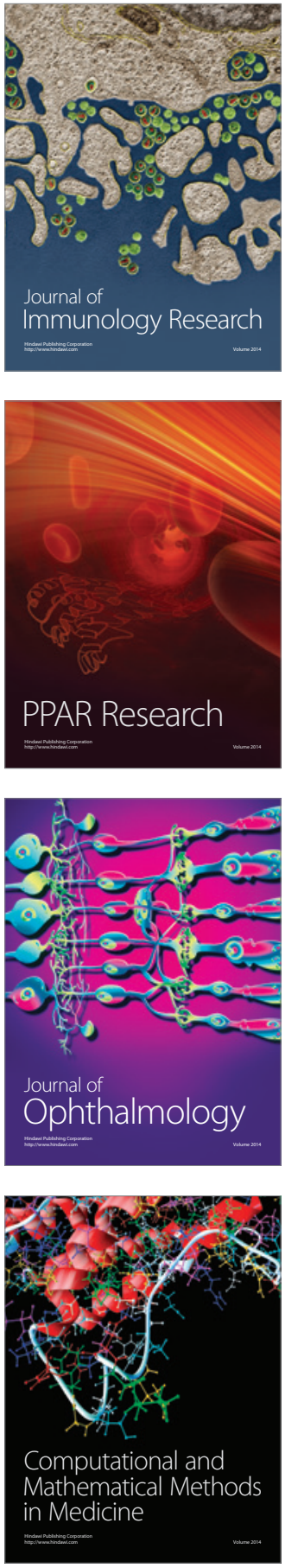

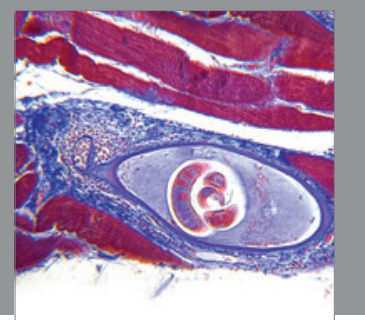

Gastroenterology

Research and Practice
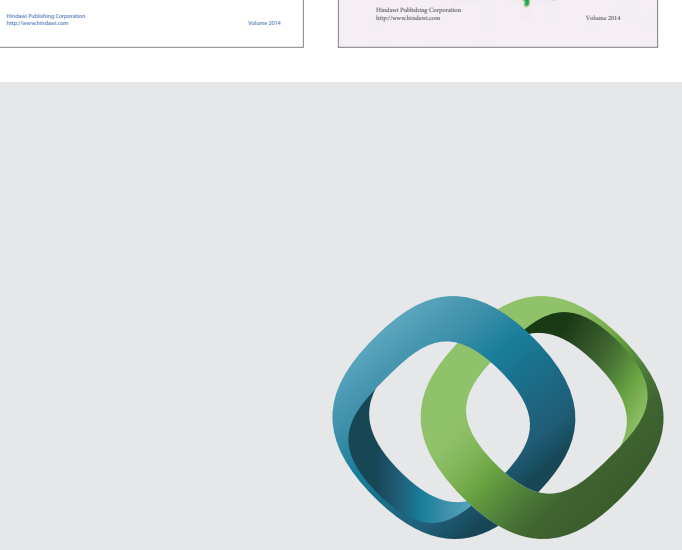

\section{Hindawi}

Submit your manuscripts at

http://www.hindawi.com
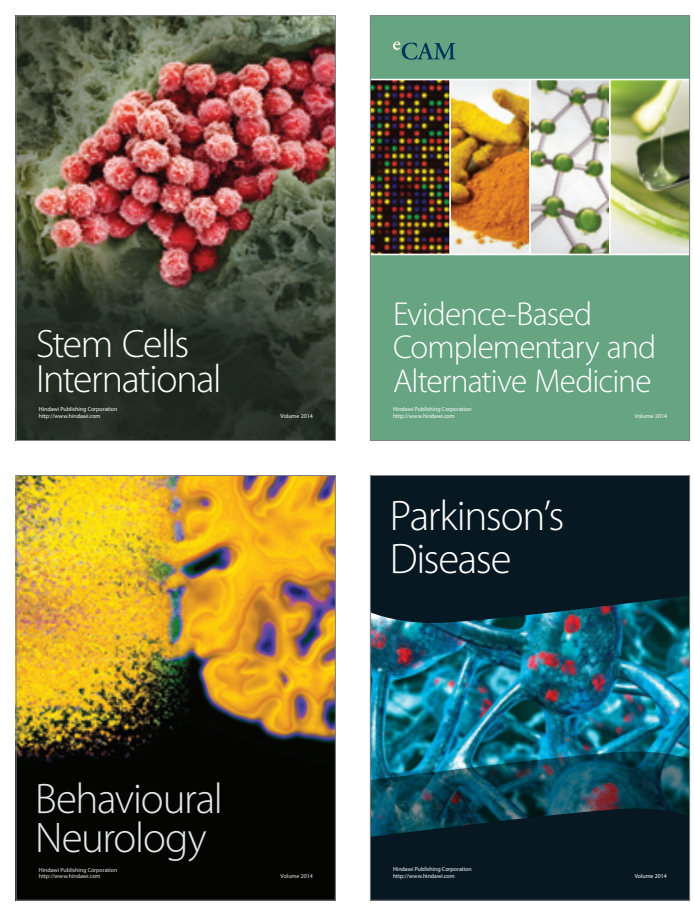

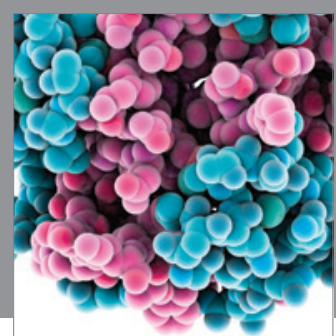

Journal of
Diabetes Research

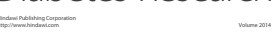

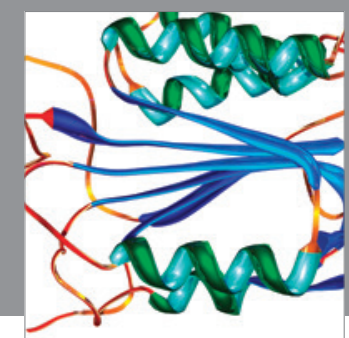

Disease Markers
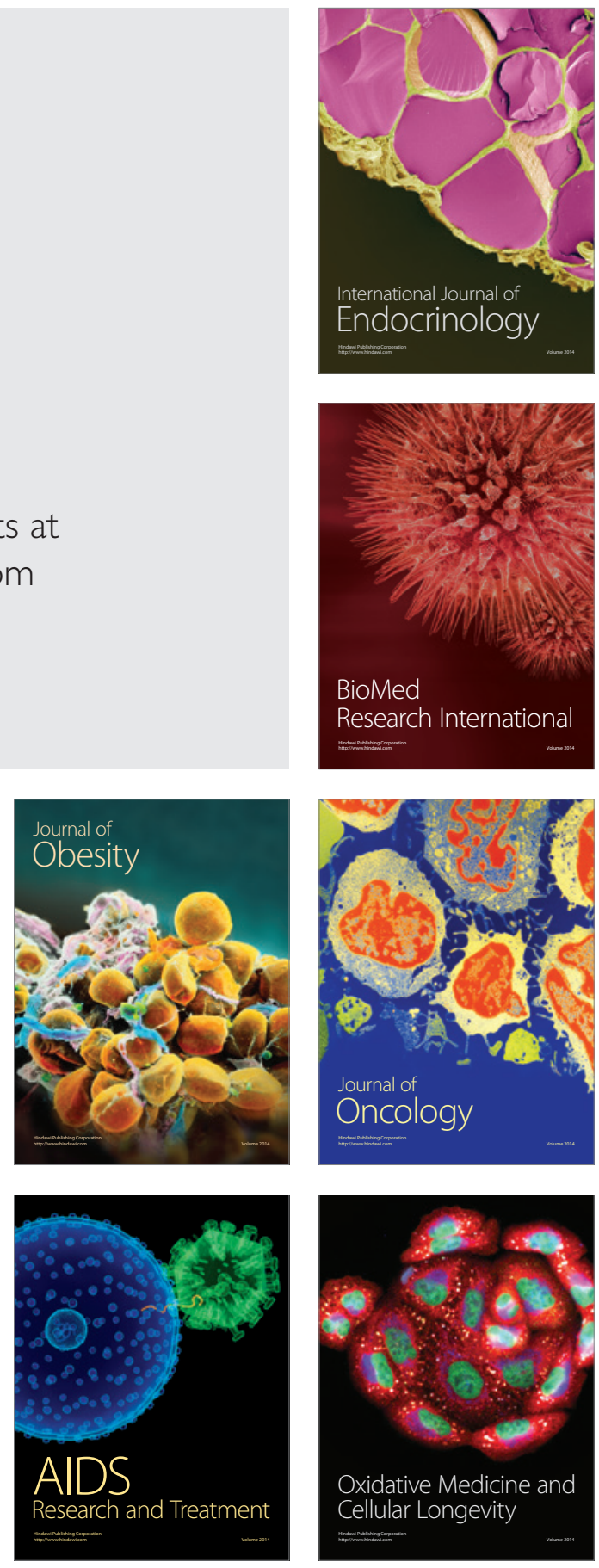